\title{
Bacterial quorum sensing and biofilm formation
}

Many bacteria are known to regulate their cooperative activities and physiological processes through a mechanism called quorum sensing (QS), in which bacterial cells communicate with each other by releasing, sensing and responding to small diffusible signal molecules called autoinducers ${ }^{1}$. This process of intercellular communication called quorum sensing was first described in the marine bioluminescent bacterium Vibrio fischeri ${ }^{2}$. Vibrio fischeri lives in symbiotic associations with a number of marine animal hosts. In these partnerships, the host uses light produced by Vibrio fischeri for specific purposes such as attracting prey, avoiding predators or finding a mate. In exchange for the light it provides, Vibrio fischeri obtains a nutrient-rich environment where it resides. A luciferase enzyme complex is found to be responsible for light production in Vibrio fischeri. Bioluminescence occurs only when Vibrio fischeri is at high cell density, which is controlled by quorum sensing 3 .

It is now known that many bacteria (Bacillus subtilis, Pseudomonas aeruginosa, Staphylococcus aureus, Streptococcus mutans, Streptococcus pneumonia, etc) regulate their social activities and physiological processes through a quorum sensing mechanism, including symbiosis, formation of spore, bacteriocin production, genetic competence, programmed cell death, virulence and biofilm formation ${ }^{4}$. The process controlled by quorum sensing is diverse and reflect the specific needs of particular communities. In many bacteria, quorum sensing represents a central mechanism to regulate social activities, allowing bacteria to reap benefits that would be unattainable to them as individual cells. Increasing evidence shows that quorum sensing mediated social activities favor microbial interactions and are believed as major mechanisms to regulate population level virulence of bacteria. These studies have produced important insights into the social biology of microbes in biofilms and in bacterial infections ${ }^{5,6,7}$.

Quorum sensing is emerging as an integral component of bacterial global gene regulatory networks responsible for bacterial adaptation in biofilms. The discovery of the widespread use of quorum sensing system in bacteria is pivotal in guiding researchers to study bacterial multicellular behaviors rather than the previous emphasis on individual cell biological processes. However, research on how bacterial quorum sensing works mechanistically in biofilms remain in their infancy. A clear challenge facing the field is to determine what factors of a biofilm influence the onset of quorum sensing and subsequent gene expression ${ }^{8}$. Another key challenge is to determine functional consequences of quorum sensing in multi-species biofilms. The answer to these questions will undoubtedly provide new insights and surprises.

\section{References:}

1. Miller M B, Bassler B L. Quorum sensing in bacteria. Annu. Rev, Microbiol. 2001;55:165-199

2. Waters C M, Bassler B L. Quorum sensing : cell-to-cell communication in bacteria. Annu. Rev. Cell. Dev. Biol. 2005;21:319-346

3. van Bodman S B, Willey J M, Diggle S P. cell-cell communication in bacteria: united we stand. J Bacteriol. 2008;190;4377-4391

4. Nadell C D, Xavier J B, Foster K R. the sociobiology of biofilms. FEMS Microbiol. Rev. 2009;33:206-224

5. Cvitkovitch D G, Li Y H, Ellen R P. Quorum sensing and biofilm formation in Streptococcal infections. J. Clin. Invest. 2003;112:1626-1632

6. Juhas M, Eberl L, Tummler B. Quorum sensing: The power of cooperation in the world of Pseudomonas. Environ Microbiol.2005;7:459-471

7. Antunes L C, Ferreira R B, Buckner M M Finlay B B. Quorum sensing in bacterial virulence. Microbiology. 2010;156: 2271-2282.

8. Quorum sensing and bacterial social interactions in biofilms. Sensors. 2012;12: 2519-2538.

\section{Prof. Ahmed Abu Saleh}

Department of Microbiology and Immunology BSMMU; Shahbag, Dhaka

aasaleh@gmail.com 